\title{
The role of glyclinergic interneurons in the dorsal column nuclei
}

\author{
E. Sánchez, J. Aguilar, C. Rivadulla, A. Canedo
}

\begin{abstract}
The aim of this paper is to provide new insights about the circuitry and the role of the dorsal column nuclei (DCN) in processing somatosensory information. The presence of glycinergic cells, a second type of DCN interneurons in addition to well-known GABAergic interneurons, opens the door to more complex interactions between cuneate cells as well as to a new hypothesis about the computational implications of such interactions. The research posed here fits in a broader context in the field of the sensory systems and deals with the general issue on the role of subcortical structures (i.e the thalamus) in processing sensory information.
\end{abstract}

Keywords

Dorsal column nuclei; Somatosensory system; Computational models; Optimal coding

\section{Introduction}

The dorsal column nuclei (DCN) are located within the brainstem, close to the rostral area of the spinal cord, and are constituted by the gracile $(\mathrm{GN})$ and the Cuneate $(\mathrm{CN})$ nuclei. They present a similar organization, but differ on the origin of their primary afferents. The DCN process somatosensory information coming from primary afferent fibres and send it to the thalamus via the medial lemniscus (ML). The experimental data were obtained from the dorsal part of the middle region of the $\mathrm{CN}$, which is located from the obex to about $4 \mathrm{~mm}$ caudal to it in the cat. This region receives information from somatotopically organized primary afferents sensitive to both light touching or hair displacement, and distal receptive fields (RFs). On top of that, the $\mathrm{CN}$ receives an important corticofugal projection from layer $\mathrm{V}$ of sensory motor cortex [8], [13], [14] and [18], with a topographic organization similar to the primary afferents [3], [4], [6], [9], [10] and [11].

A key point to understand the cuneate function better would undoubtedly be the precise knowledge of the RF structure of the different cell types implicated in the $\mathrm{CN}$ circuitry: relay or cuneo lemniscal (CL) cells, and GABAergic (GAB) interneurons or non-CL cells. The current knowledge is limited mainly to CL cells. It has been recently shown that their RF has an excitatory center and an inhibitory surround [7]. This spatial arrangement is generated through somatotopically organized afferent organization consisting of direct excitatory input on CL cells and GAB mediated inhibition from surrounding areas [7]. It has been demonstrated that when this circuit is constructed with realistic models of cuneate cells [16], it can perform edge detection as well as motion discrimination [15].

Moreover, there exists evidence of additional GAB interneurons performing recurrent lateral inhibition [2]. GAB participation seems evident when ejection of bicuculline (BiCu) after lemniscal stimulation uncovers recurrent excitation (Fig. 1A1). The effect of recurrent inhibition is clarified when recurrent activation is blocked after increasing lemniscal stimulation to $2 \mathrm{~mA}$ (Fig. 1A2). GAB interneurons are involved in this circuitry when $\mathrm{BiCu}$ ejection (Fig. 1A2, right) imposes the activity initially found under control conditions (0.2 $\mathrm{mA}$ stimulation). 
(A)

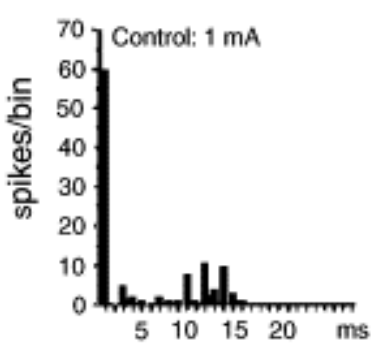

(B)

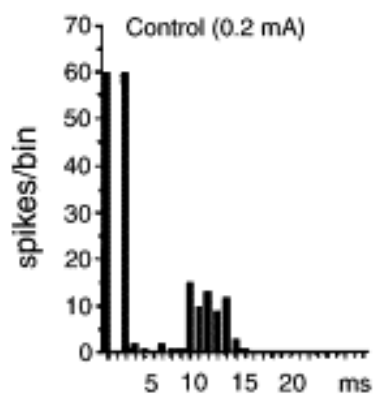

A1

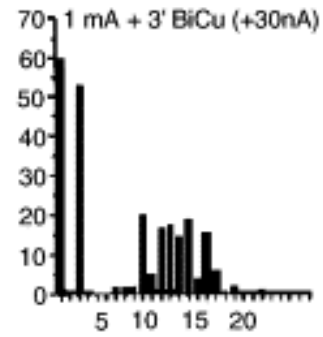

A2

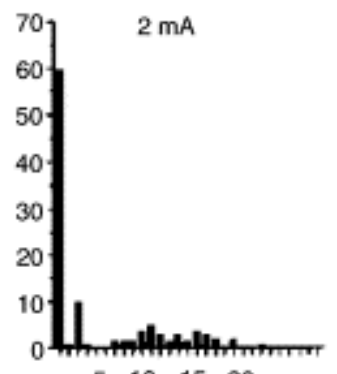

$\begin{array}{lllll}5 & 10 & 15 & 20\end{array}$

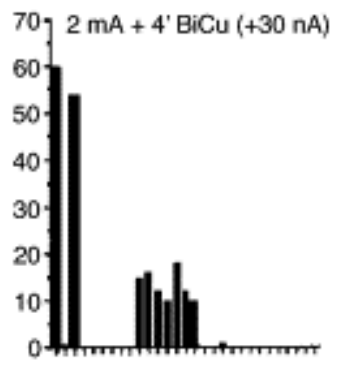

$\begin{array}{lllll}5 & 10 & 15 & 20\end{array}$

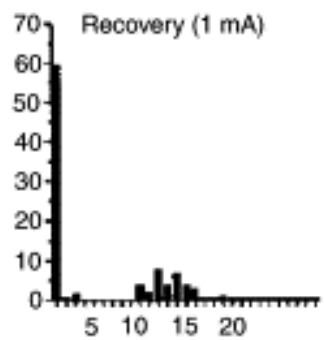

5

ML stimulation
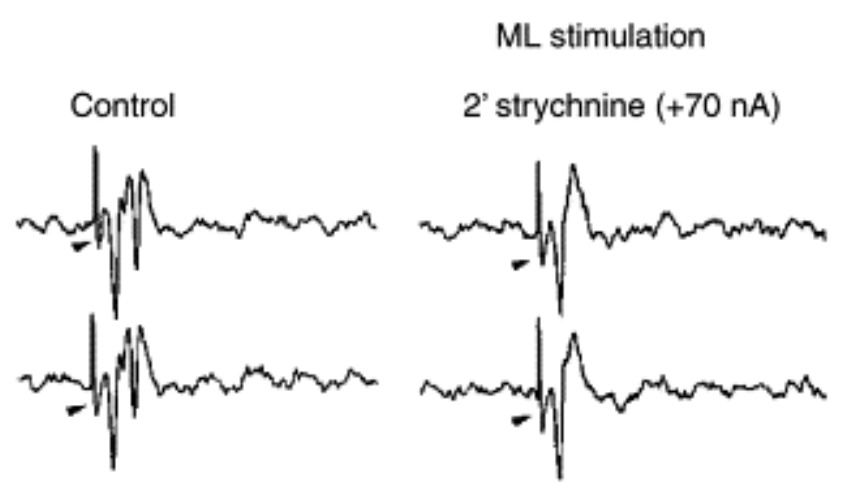

Recovery

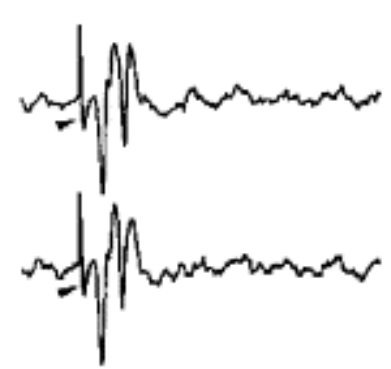

Fig. 1. Lemniscal stimulation and recurrent effects. ML stimulation and Bicuculline (GABA antagonist) ejection shows GAB neurons participating in lateral recurrent effects (A). ML stimulation and strychnine ejection uncovers inhibitory effects on CL cells (B).

At this point, the presence of a second interneuronal type, the glycinergic (GLY) interneurons, which constitute about $30 \%$ of total neuronal population in the rat [12], introduces further complexity into the circuit. It is known that GLY neurons evoke facilitation of relay cells by inhibiting GAB interneurons [1] and [2]. Fig. 1B shows that application of strychnine, a glicine antagonist, after ML stimulation, allowed the blocking of the second recurrent spike.

Unfortunately, we do not have experimental evidences related to the RF structure of these interneurons that could help us understand its role better. So, we have resorted to computational models in order to explore the role that different spatial arrangements of GLY cells could have on the processing of CL cells. 


\section{Methods}

The computational model assumes that CL cells show excitatory center-inhibitory surround RFs, in which inhibition is performed by GAB interneurons. With regard of the recurrent organization, and considering the experimental data discussed so far, we also have lateral inhibition through $\mathrm{GAB}$ interneurons over CL cells as well as a disinhibition mechanism mediated by GLY interneurons (Fig. 2).

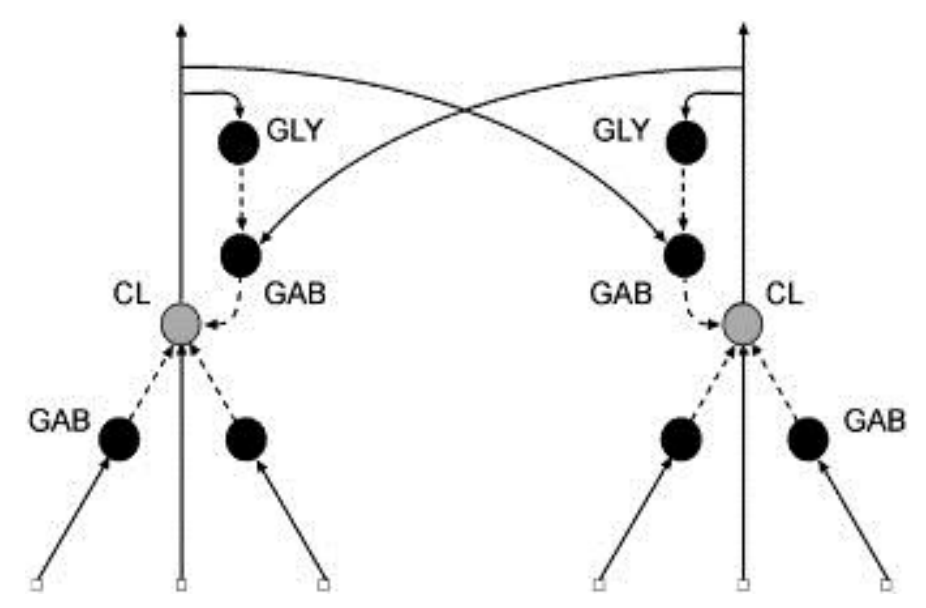

Fig. 2. A model of the $\mathrm{CN}$ circuitry. CL neurons have both afferent and recurrent influences. GAB neurons (GAB) seem to be involved in afferent inhibition as well as recurrent lateral inhibition. GLY interneurons perform disinhibition over CL cells by inhibiting GAB interneurons.

For the simulation, we have implemented a bidimensional version of the circuitry in which all neurons have a square-shaped RF. Under this configuration, CL neurons have an afferent RF, which is constituted by excitatory input coming from the receptors at the center of the square and inhibitory input from the surrounding edges, and a recurrent inhibitory contribution from corresponding GAB interneurons. These ones receive excitatory input only from CL cells with non-overlapped RFs. Finally, GLY interneurons show a fully excitatory RFs coming from recurrent collaterals of CL cells with overlapped RF.

As we focus on circuit behavior, we have simplified the neuronal models. The neurons are McCulloch-Pitts units, in which the output of the $j$ th neuron is $y_{j}=\Psi\left(\Sigma w_{j i} x_{i)}\right.$ with the activation function $\Psi$ being the threshold type. Contribution of synapse between neuron $i$ th and neuron $j$ th is modelled through weight $w_{j i}$. Weights and RF configurations are the most relevant free parameters in order to investigate the processing role of each neuronal type.

\section{Results}

We have built and tested the circuit step-by-step, starting with the afferent connections, and iteratively adding the recurrent GAB and GLY layers. Fig. 3 shows how a layer of CL neurons can perform edge detection based on the afferent center-surround organization. CL cells having object edges at the center of their RFs show a higher activity (black) than those cells receiving input from the inner parts (grey). We have found that the edge detection is quite robust against size, form and also intensity of the stimulus. However, in order to achieve robustness against stimulus intensity, a gain control mechanism like, for instance, shunting inhibition, seems to be required. As this is a problem out of the scope of this paper, the mechanism was not shown in Fig. 2. 


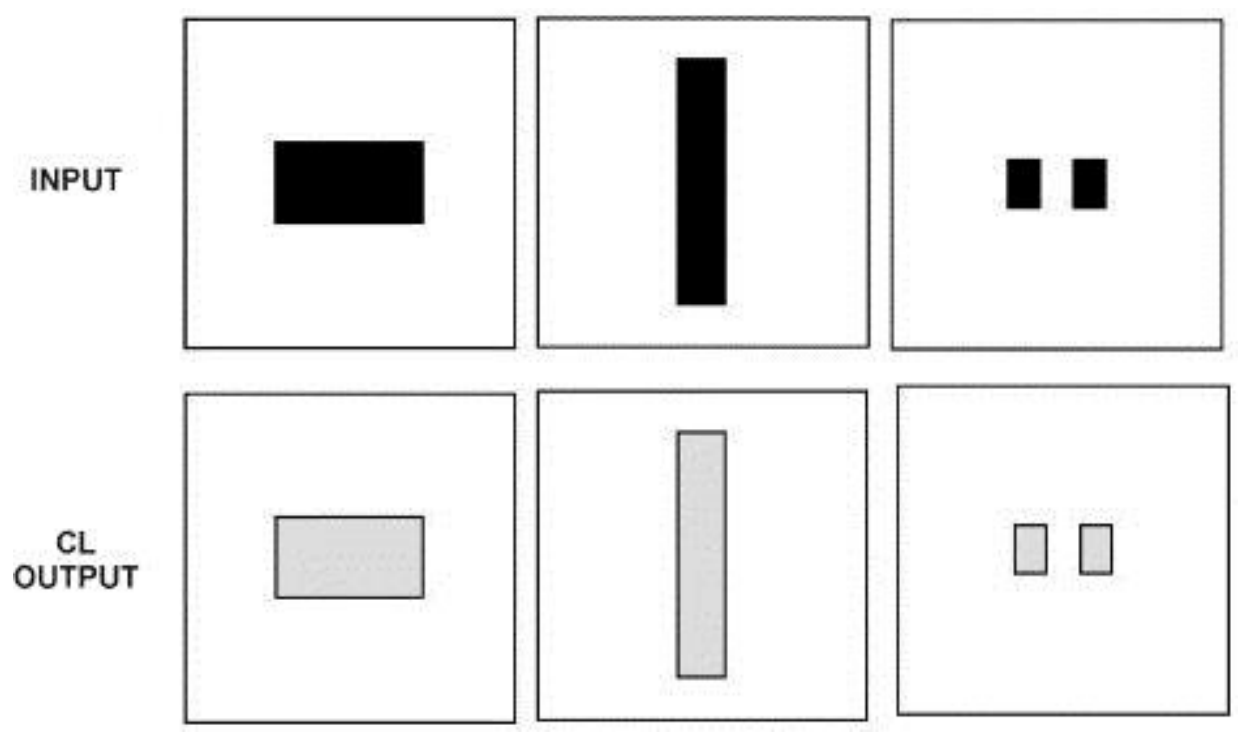

Fig. 3. Edge detection through afferent connections. Inputs of different forms and sizes were presented (top). CL cells (bottom) respond most remarkably to edges (black) than other parts of the objects (grey).

Recurrent lateral inhibition was then added to the circuitry (Fig. 4), with an RF size of 9×9. After the first iteration, in which regular edge detection is performed, the output shows an oscillatory activity consisting of two states: in the first one, object edges are detected; in the second one, it is the middle region of the object that is highlighted. This result could be understood as an optimal spatial coding as CL output encodes the most relevant information: (1) edge location, and (2) that the object is of a solid type.

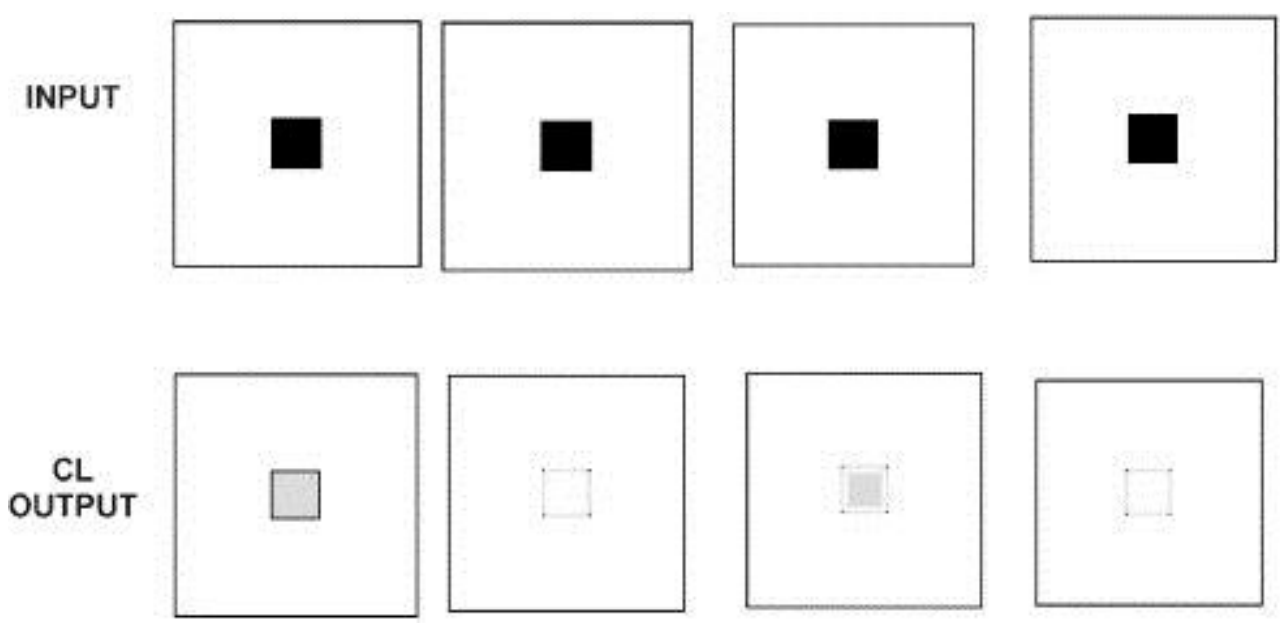

Fig. 4. Afferent connections and recurrent lateral inhibition. The output shows an oscillatory pattern between two states that encode the edges and the object's middle region.

The encoding is robust against lateral inhibition strength, i.e. the value of synapse weights between CL neurons and GAB interneurons. However, the effect depends on the GAB interneurons RF. When RF has a size bigger than $21 \times 21$, the two-state oscillation disappears and the output seems to enter a chaotic pattern.

The most interesting result is obtained when GLY interneurons are introduced (Fig. 5). The size of GLY RF was initially set to $3 \times 3$. The output evolves from an initial state, where edges are detected, into a fill-in process that finishes with the complete covering of the object (Fig. 5). The bigger the GLY RF, the 
higher the speed of the whole process. As it happened with the GAB layer and the spatial coding, this temporal coding effect is also robust against inhibition strength.

1

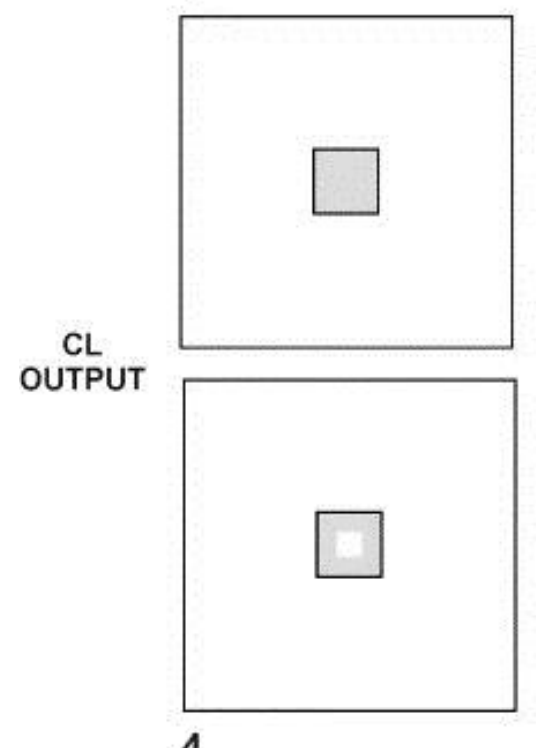

4
2
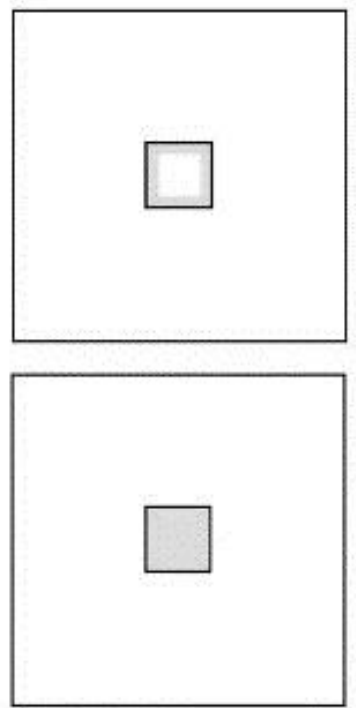

5
3
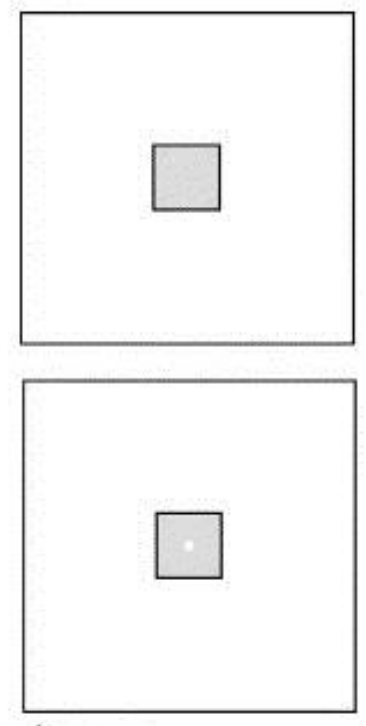

6

Fig. 5. CL outputs with the complete circuitry. Iterations (1-6) indicate how CL output evolves with time.

The fill-in process could be explained by considering the activity carried out in both GAB and GLY maps. The GAB map presents a final state in which the object's edges are represented, while the GLY map finally represents the object's size. So it seems that CL output is caused by the interplay between the lateral recurrent inhibition (GAB map) and the recurrent disinhibition (GLY map).

\section{Discussion}

The CN processing shown in Fig. 5 can be understood as an optimal spatio-temporal coding. Spatial coding is clearly performed in Fig. 4, in which the most salient regions of the object (edges and middle zone) are detected. On top of that, an optimal temporal coding, introduced by GLY neurons, allows a progressive transmission of information up to the ventro-postero-lateral (VPL) nucleus in the thalamus. It starts with high-level informative regions (edges, Fig. 5-2) and finishes with low-level informative regions (core middle zone, Fig. 5-6). This behavior presents certain similarities with the popular JPEG compression algorithm, which is responsible for the progressive fill-in of images in web browsers. On each domain, both VPL and the user might stop the transmission of information when the available information is sufficient enough to identify the incoming patterns.

There is an issue that concerns as to why the whole object appears interleaved between the fill-in stages (Fig. 3, Fig. 4 and Fig. 5). There is an experimental evidence that VPL neurons respond with a much higher reliability to a number of consecutive spikes than to single spikes [17]. Considering this, we can claim that: (1) single spikes generated from CL cells related to the middle region of the object (Figs. 5-1, 5-3 and 5-5) might not generate any activity in VPL, (2) the interleaved pattern ensures that VPL will start firing just when CL cells start to generate spike trains as a result of the fill-in process.

The $\mathrm{CN}$ processing discussed so far supports the thesis that the nervous system is designed to detect changes in the environment, discard those signals that do not contribute with relevant information, and encode it in an optimal manner [5]. 


\section{References}

[1]. J. Aguilar, C. Rivadulla, C. Soto, A. Canedo. New corticocuneate cellular mechanisms underlying the modulation of cutaneous ascending transmission in anesthetized cats. J. Neurophysiol., 89 (2003), pp. 3328-3339.

[2]. J. Aguilar, C. Soto, C. Rivadulla, A. Canedo. The lemniscal-cuneate recurrent excitation is suppressed by strychnine and enhanced by GABAA antagonists in the anaesthetized cat. Eur. J. Neurosci., 16 (2002), pp. $1697-$ 1704.

[3]. V.E. Amassian, J.L. De Vito. La transmission dans le noyau de Burdach (nucleus cuneatus), Etude analytique par unités isolées d'un relais somatosensoriel primaire. Coll. Int. Centre. Nat. Rech. Sci., 67 (1957), pp. 353-393.

[4]. P. Andersen, J.C. Eccles, R.F. Schmidt, T. Yokota. Depolarization of presynaptic fibres in the cuneate nucleus. J. Neurophysiol., 27 (1964), pp. 92-106.

[5]. H.B. Barlow. The coding of sensory messages. W.H. Thorpe, O.L. Zangwill (Eds.), Current problems in animal behaviour, Cambridge University Press, Cambridge (1961), pp. 330-360.

[6]. K.J. Berkley, R.J. Budell, A. Blomqvist, M. Bull. Output systems of the dorsal column nuclei in the cat. Brain. Res. Rev., 11 (1986), pp. 199-225.

[7]. A. Canedo, J. Aguilar. Spatial and cortical influences exerted on cuneothalamic and thalamocortical neurons of the cat. Eur. J. Neurosci., 12 (2000), pp. 2515-2533.

[8]. W.W. Chambers, C.N. Liu. Cortico-spinal tract of the cat, An attempt to correlate the pattern of degeneration with deficits in reflex activity following neocortical lesions. J. Comp. Neurol., 108 (1957), pp. 23-55.

[9]. S. Cheema, B.L. Whitsel, A. Rustioni. The corticocuneate pathway in the cat: relations among terminal distribution patterns, cytoarchitecture, and single neuron functional properties. Somatosen Res., 1 (1983), pp. 169-205.

[10]. R.W. Dykes, D.D. Rasmusson, D. Sretavan, N.B. Rehman. Submodality segregation and receptive-field sequences in cuneate, gracile, and external cuneate nuclei of the cat. J. Neurophysiol., 47 (1982), pp. 389-416.

[11]. G. Gordon, W.A. Seed. An investigation of the nucleus gracilis of the cat by antidromic stimulation. J. Physiol. (London), 155 (1961), pp. 589-601.

[12]. J.H. Lue, S.M. Luai, T.J. Wang, J.Y. Shieh, C.Y. Wen. Synaptic relationships between corticocuneate terminals and glycine-immunoreactive neurons in the rat cuneate nucleus. Brain Res., 771 (1997), pp. 167-171.

[13]. L. Martinez, J.A. Lamas, A. Canedo. Pyramidal tract and corticospinal neurons with branching axons to the dorsal column nuclei of the cat. Neuroscience, 68 (1995), pp. 195-206.

[14]. A. Rustioni, N.L. Hayes. Corticospinal tract collaterals to the dorsal column nuclei of cats. Exp. Brain Res., 43 (1981), pp. 237-245.

[15]. E. Sánchez, S. Barro, A. Canedo. Edge detection and motion discrimination in the cuneate nucleus. Lect. Notes Comp. Sci., 2415 (2002), pp. 198-203.

[16]. E. Sánchez, S. Barro, J. Mariño, A. Canedo. A computational model of cuneothalamic projection neurons. Network Comput. Neural Syst., 14 (2003), pp. 211-231.

[17]. W.M. Usrey, J.B. Reppas, R.C. Reid. Paired-spike interactions and synaptic efficacy of retinal inputs to the thalamus. Nature, 395 (1998), pp. 384-387.

[18]. F. Walberg. Corticofugal fibres to the nuclei of the dorsal columns. An experimental study in the cat. Brain, 80 (1957), pp. 273-287. 